\title{
Beam tracing study for design and operation of two-pass electron cyclotron heating at ASDEX Upgrade
}

\author{
Martin Schubert ${ }^{1,}$, Burkhard Plaum $^{2}$, Jörg Stober ${ }^{1}$, Albrecht Herrmann ${ }^{1}$, Walter Kasparek ${ }^{2}$, Carsten Lechte ${ }^{2}$, \\ Fritz Leuterer ${ }^{1}$, Francesco Monaco ${ }^{1}$, Bernhard Petzold ${ }^{1}$, Emanuele Poli ${ }^{1}$, Stefan Vorbrugg ${ }^{1}$, and Dietmar Wagner ${ }^{1}$ \\ ${ }^{1}$ Max Planck Institute for Plasma Physics, Boltzmannstr.2, 85748 Garching, Germany \\ ${ }^{2}$ Institut für Grenzflächenverfahrenstechnik und Plasmatechnologie, Universität Stuttgart, \\ Pfaffenwaldring 31, 70569 Stuttgart, Germany
}

\begin{abstract}
The electron cyclotron resonance heating system at ASDEX Upgrade (AUG) is currently being extended to eight similar Gyrotrons in total. Each Gyrotron operates at 105 and $140 \mathrm{GHz}$ and is designed for up to $1 \mathrm{MW}$ millimetre wave output power. A substantial part of the AUG program will focus on experimental conditions, where the plasma density may be above the X-2 cut-off density at $140 \mathrm{GHz}$. In order to cope with the high density, the heating system will operate in the O-2 mode scheme with potentially incomplete absorption in the first pass. Reflecting gratings installed into the heat shield on AUG's inner column allow for a controlled second pass of the beam's unabsorbed fraction. Thermocouple measurements serve to control the beam position on the grating. The beam geometry is being finalized for the launchers \#1-4. Beam propagation is simulated with the TORBEAM code and previous high density experiments are used as a database. The geometry is optimized using three criteria: central deposition, high absorption and robustness of the beam dump after the second pass. The experimental conditions, and the plasma electron density in particular, may vary such that the Gaussian beam parameters of the incoming beam on the grating deviate from the design values. It is proposed to model the effect of the grating with an equivalent ellipsoidal mirror. Laboratory measurements are shown, which support this model.
\end{abstract}

\section{Introduction}

Plasma electron densities up to $1 \cdot 10^{20} \mathrm{~m}^{-3}$ are regularly achieved in ASDEX Upgrade (AUG) experiments. Examples are high heat flux experiments [1] and fuelling with pellets [2]. In the latter case the pellet ablation temporary and locally increases the plasma electron density even further. With respect to the electron cyclotron resonance heating (ECRH) system, operating at $140 \mathrm{GHz}$, the density may exceed the X-2 cut-off density of $1.2 \cdot 10^{20} \mathrm{~m}^{-3}$. A robust countermeasure is to change the launched polarisation at the given second harmonic to O-Mode (O-2), which doubles the cut-off density. Due to the finite absorption [3], there are several important constraints on the O-2 scheme: In a situation typical for an AUG experiment, the maximum optical thickness $\tau$ is around 1 (figure 1). This corresponds to approximately one third of the launched beam power leaving the plasma, the so-called 'shine-through', after the first pass. Moreover, the maximum $\tau$ is only obtained with a beam injection angle oblique to the magnetic field vector. Deviations between $10^{\circ}$ and $30^{\circ}$ from the perpendicular direction are necessary (figure 1). The optical thickness $\tau$ scales as $T_{\mathrm{e}}^{2}$ [3]. The electron temperature $T_{\mathrm{e}}=4 \mathrm{keV}$ was used in figure 1 . If $T_{\mathrm{e}}$ is significantly smaller, then $\mathrm{O}-2$ mode heating might not be applicable due to lack of efficiency.



Fig. 1. Optical thickness $\tau$ of a model plasma for the second harmonic O-mode, function of the plasma electron density $n_{\mathrm{e}}$ according to [3]. This was evaluated for the launch angles $40^{\circ}$, $60^{\circ}, 80^{\circ}$ and $90^{\circ}$ between $\mathbf{k}$ and $\mathbf{B}$, and using parameters $B_{0}$, $R_{0}, T_{\mathrm{e}}, f$ of a typical ASDEX Upgrade experiment.

Even at nominal conditions at AUG, if one assumes a typical launched millimetre wave power of up to $900 \mathrm{~kW}$ with a compact beam diameter of a few centimetres and with the described plasma absorption properties, then the shine-through on the high field side corresponds to a power density on plasma facing components, which may 
cause damage [4]. It was proposed $[5,6]$ to install a reflector at the position of the shine-through, which allows the unabsorbed fraction to make a controlled second pass through the plasma. In the design it should be taken into account that the second pass ends on a robust vessel component. The remaining unabsorbed beam power typically does not exceed $10 \%$ of the launched power and further beam propagation is usually not taken into account.

The two-pass heating scheme has exemplarily been demonstrated before [6]. With the persisting demand for ECRH in AUG experiments and with the on-going modernization of major ECRH components [7], it was decided to equip all launchers with the O-2 mode capability. This paper introduces the two-pass heating design for the launchers \#1-4 in section 2. Beam propagation and absorption were simulated using the TORBEAM code [8].

The mirror gratings [5], which are used as reflectors, are designed and optimized for a given operating point. If the plasma parameters and the plasma electron density in particular are modified, then beam path and beam shape are changing. In section 3 it is proposed to model the reflection on the mirror grating in such case with an equivalent ellipsoidal mirror. This is tested in a laboratory mock-up of the reflection grating with a 140 $\mathrm{GHz}$ source and network analyser.

\section{Electron cyclotron heating at AUG}

With the modernization of AUG's first ECRH system, it is planned to have a final stage with 8 dual frequency $(105 / 140 \mathrm{GHz})$ Gyrotrons [7] with up to $1000 \mathrm{~kW}$ millimetre wave output power per Gyrotron. During the last 2017 experimental campaign, 6 Gyrotrons were operational. Inside AUG, the millimetre wave power is launched at distinct positions (figure 2) and the beam direction can be controlled with movable mirrors [7]. Unabsorbed millimetre wave power is monitored with a set of detectors [9], including the AUG ECE system, cf. figure 2. All detectors are part of a fast interlock system, which can shut down Gyrotron operation in the case of a stray radiation excess.

The particularity of the O-2 mode shine-through is exemplarily visualized in figure 3 for AUG launcher \#5. It is compared with the frequently used X-2 scheme in the same figure using launcher \#8. The X-2 scheme has a nearly perfect absorption for a broad range of plasma parameters and launching geometries [3]. It is, however, only applicable for densities up to $1.2 \cdot 10^{20} \mathrm{~m}^{-3}$ at perpendicular incidence. The cut-off density can be significantly smaller, if the beam injection deviates from the perpendicular direction [3].

\subsection{First pass and reflector position}

The first step of the design is to determine the reflector position for a given launcher. Different positions are tested by simulating the beam path and the first pass absorption with the TORBEAM code [8] in the same model plasma. Depending on the position, the launch angles have to be adapted in order to make sure that the shine through beam after the first pass ends centrally on the reflector. Maximum possible absorption (section 1) depends on the angle between $\mathbf{k}$ and $\mathbf{B}$, which is mainly determined by the toroidal launch angle. In our model plasmas, high first pass absorption correlates well with central power deposition. This is due to the generally peaked temperature profiles, the strong $T_{\mathrm{e}}$ dependence of the O-2 absorption, and the alignment of the absorption zone with the magnetic axis.

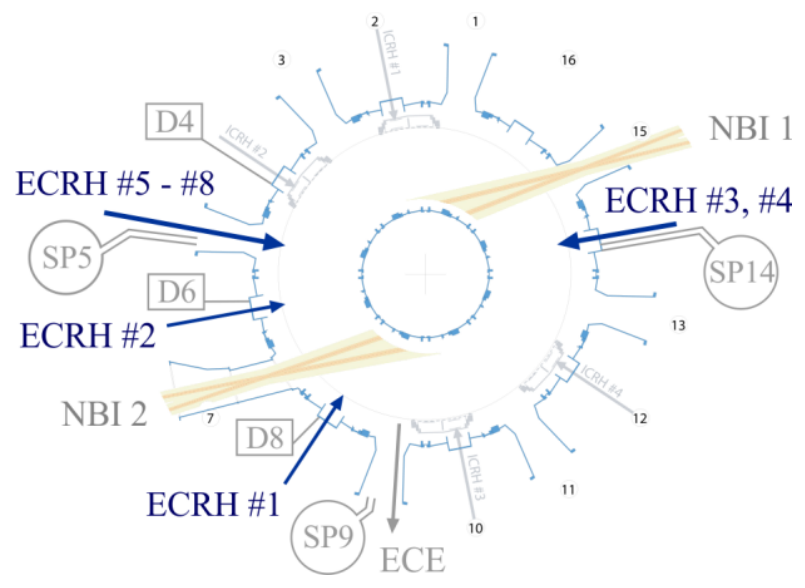

Fig. 2. Top view of the equatorial cross section through AUG with launching positions of different heating systems. ECRH launchers \#5 - \#8 are operational, incl. O-2. ECRH \#1 and \#2 are operational, launchers $\# 3$ and $\# 4$ are currently being upgraded. O-2 is being designed for launchers \#1 - \#4. Relevant detectors for unabsorbed radiation are shown in light colour. 'SP' Sniffer sphere, 'D' D-band pick-up waveguide, mounted on quartz vacuum window $[4,10]$.



Fig. 3. Vertical cross section at the ECRH \#5 - \#8 launching position, visualising the $140 \mathrm{GHz}$ beam path in a typical AUG experiment $\left(B_{0}=2.5 \mathrm{~T}, T_{\mathrm{e}}=4 \mathrm{keV}\right)$ with different polarisation. Upper launcher: ECRH \#5 with O-mode, lower launcher: ECRH \#8 with X-mode polarisation. 


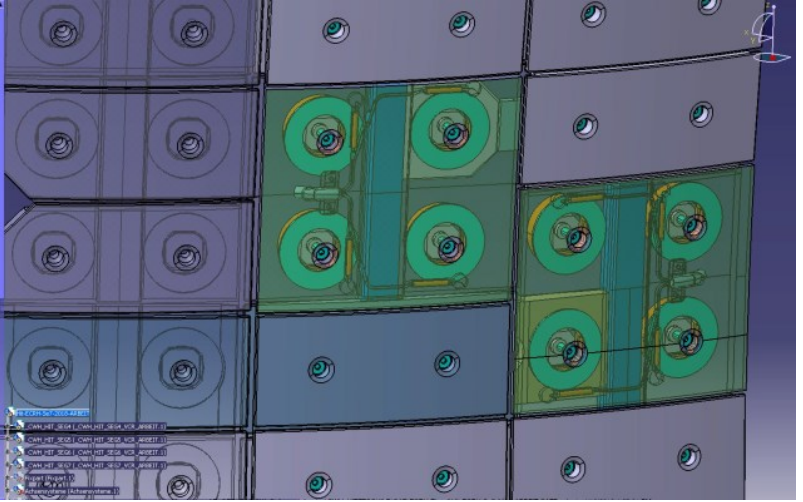

Fig. 4. Tiles of the AUG heat shield with double tile models for the reflectors in a CAD drawing. The left column is transparent in order to visualise the underlying support structure. Both reflector double tiles (middle and right column) are transparent to show the built-in thermocouple measurement.

TORBEAM input data were taken from AUG $\# 30505, \mathrm{t}=3.3 \mathrm{sec}$, considered a model high power discharge with partial detachment. Characteristic data: 19.5 MW total heating power, $I_{\mathrm{p}}=1.2 \mathrm{MA}, B_{\mathrm{t}}=-2.48 \mathrm{~T}$, $n_{\mathrm{e}}=1.15 \cdot 10^{20} \mathrm{~m}^{-3}$, and central $T_{\mathrm{e}}=3.6 \mathrm{keV}$. The beam path may vary with different model plasmas. Given the alignment of the absorption zone with the magnetic axis is similar, then this variation, however, is generally small when compared to the variation that corresponds to different reflector positions.

Only those reflector positions are allowed, which fit into the heat shield on the central column inside AUG (figures 3 and 4). The heat shield consists of tiles, each with the dimensions of approximately $210 \mathrm{~mm}$ (width) and $90 \mathrm{~mm}$ (height). This is the 'grid' for possible reflector positions. Two adjacent tiles (vertically) are merged, such that the reflector has the approximate dimensions $210 \mathrm{~mm} \times 180 \mathrm{~mm}$, which is considered sufficient for a good coverage of the width of a $140 \mathrm{GHz}$ ECRH beam inside AUG. The resulting 'double tiles' are exemplarily shown in figure 4 .

\subsection{Second pass and beam dump}

If the reflector has a high efficiency (section 3) and assuming similar values of the optical thickness for the first and second pass, then the unabsorbed power after the second pass will be of the order $7-8.5 \%$ of the initially launched power (figure 5). This depends on $T_{\mathrm{e}}$. The unabsorbed power may leave the plasma as a compact beam on the low field side. Hence one should take care that the in-vessel component, which faces this beam is robust enough. Typical values are $80 \mathrm{~kW}$ for the beam power and a beam radius of $5 \mathrm{~cm}$ corresponding to an area of the order of $80 \mathrm{~cm}^{2}$ and $1 \mathrm{~kW} \mathrm{~cm}$ on average. If the component has a metallic surface, then a large part of the beam will be reflected again. Usually the phase front is defocussing and with the diverging beam the power density goes down rapidly. Further propagation is, therefore, not taken into account.

In the vicinity of ECRH launchers \#1-4 there are auxiliary low field side limiters (e.g. figure 6 ) which are able to withstand the given power density. The end point of the second path for each launcher was chosen such that, according to the model beam tracing, each beam leaving the plasma is horizontally centred on the auxiliary limiter next to the launcher.

The toroidal launch angle for the second pass is determined by the position of the auxiliary limiter. For different vertical launch angles, high second pass absorption correlates with the beam passing through the plasma centre. This is similar to the first pass. According to figure 5, controlled central deposition of typically $90 \%$ of the launched power is possible.

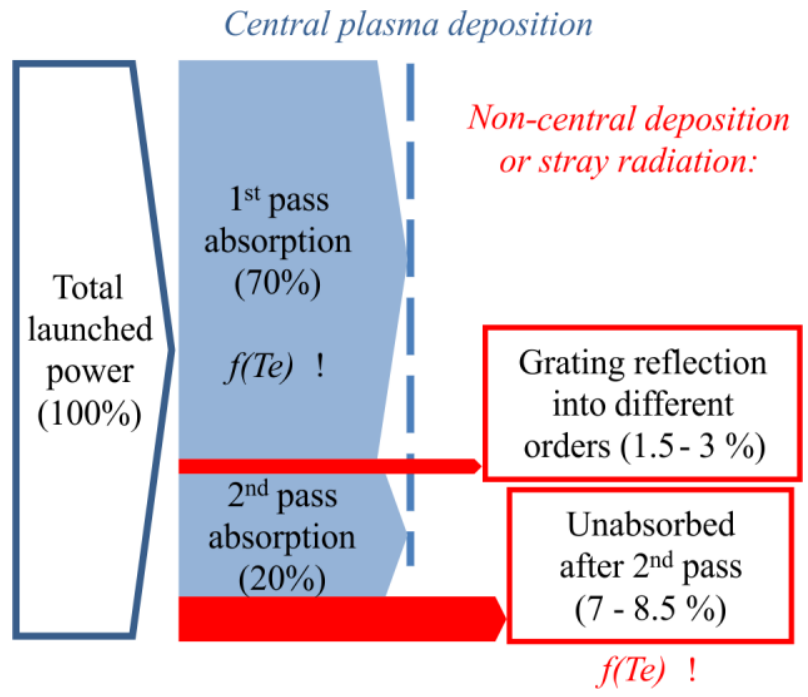

Fig. 5. Schematic power flow for the two-pass heating. These values are obtained on average with the different launching positions and the model absorption in AUG \#30505 $\mathrm{t}=3.3 \mathrm{~s}$. Both $1^{\text {st }}$ and $2^{\text {nd }}$ pass absorption depend on $T_{\mathrm{e}}$. The given numbers for the unabsorbed power are upper limits for the stray radiation, since it can be partly absorbed by the plasma.

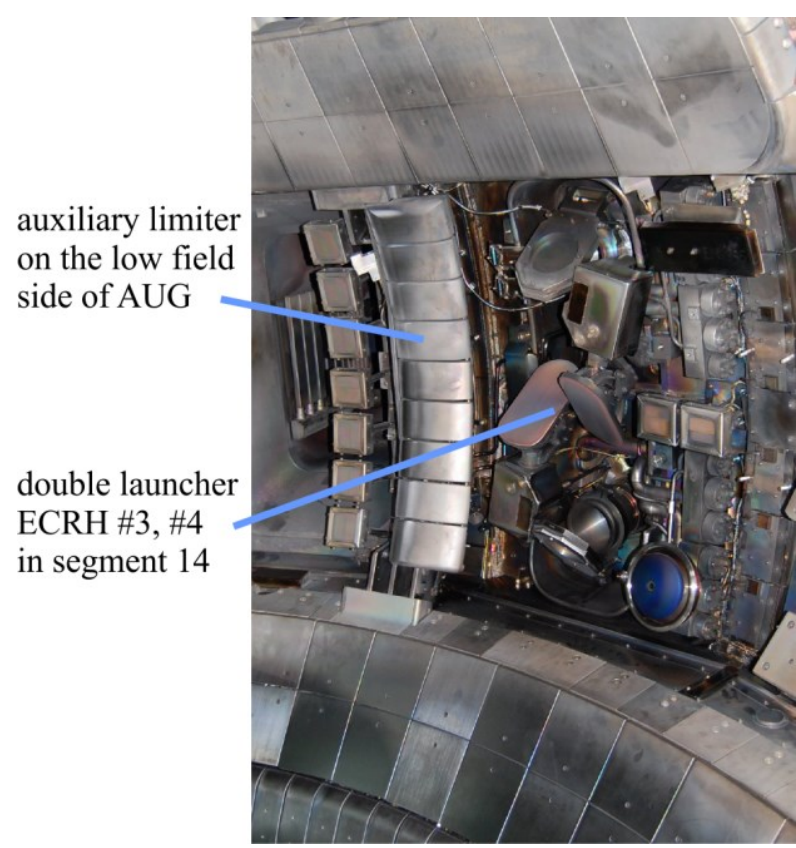

Fig. 6. Photo of the double launcher ECRH \#3, \#4 together with the auxiliary low field side limiter, both located in segment 14 . The auxiliary limiter will act as 'beam dump' for the ECRH power which is unabsorbed after the $2^{\text {nd }}$ pass. 




Fig. 7. AUG vertical cross section at the position of ECRH \#1 to visualize the TORBEAM [8] results for the two-pass heating design. In this paraxial approximation, each beam is characterized by three lines: one for the centre of the beam and at each side one for the actual 1/e width of the Gaussian.

In order to avoid depolarization between the first and the second pass, the difference of the toroidal launch angles should be small. Normally this is achieved, if the toroidal distance between launcher and beam dump is small. The reflecting grating is optimized in order to preserve the polarisation state (section 3 ).

Beam tracing results for the two-pass heating design are exemplarily shown in a vertical cross section for ECRH \#1 in figure 7. All launchers ECRH \#1-4 are located in the midplane $(\mathrm{z}=0)$, the vertical cross sections of the corresponding beam tracing are, therefore, similar. The reflecting gratings (section 3 ) offer the choice of the refocussing properties. This is a free parameter in the two-pass design. Presently, we are aiming for similar focussing properties of the first and second pass beams.

An overview of the two-pass design for ECRH \#1-4 is shown in figure 8 in a top view of the equatorial cross section through the AUG vacuum vessel. All toroidal launch angles for the first pass deviate between 12 and $15 \mathrm{deg}$ from the perpendicular direction, maximizing the first pass plasma absorption. We note that in segment 14 both ECRH \#3 and \#4 have to share the same auxiliary limiter as the end point after the second pass. While for ECRH \#1 and \#2 the decision on the geometry is final, the decision on ECRH \#4 is not yet taken. This may also have an impact on the geometry of ECRH \#3.

Altogether and with respect to the toroidal launch angles and the optical depths, the design of the two-pass heating scheme for ECRH \#1-4 follows closely the scheme for ECRH \#5-8 [6, 10]. The latter has already been proved to be reliable in AUG high density experiments. The scheme for ECRH \#1-4 features now horizontal beam paths. We would like to point out that the present two-pass design has a fixed geometry, corresponding to a fixed operating point. Large movements of the launcher mirrors are not allowed in this scheme unless additional reflectors are installed.

The intended thermocouple measurement positions on the reflecting gratings are shown (e.g. figures 4, 10c). These measurements are valuable for beam position verification and control [10].

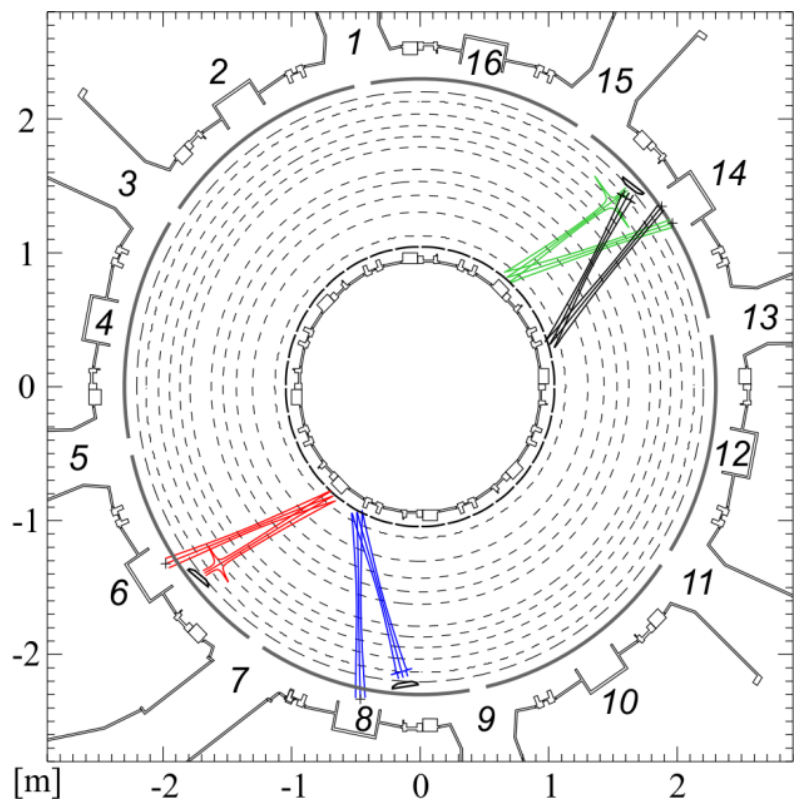

Fig. 8. Top view of the equatorial cross section through the AUG vacuum vessel, which consists of 16 segments. The outermost thin dashed line represents the plasma boundary on the low field side. The innermost thick dashes with the narrow gaps represent the tiles of the heat shield on the high field side. ECRH \#1-4 launching positions are marked with a '+' sign and to the right of each launcher the midplane cross sections of the nearest auxiliary limiters are shown. The beam tracing according to the two-pass design is overplotted using colours. Segment 8: ECRH \#1, blue. Segment 6: \#2, red. Segment 14: $\# 3$ in black and \#4 in green.

\section{Reflecting gratings}

The development of highly efficient reflecting gratings was driven by the need to reflect the beam off the tiles of the AUG heat shield (section 2.1), which results in an oblique angle between tile surface and beam direction. Both the incoming and the outgoing beam properties are design parameters and depend on the relative positions of launcher, reflector and beam dump, as well as on the beam refraction in the plasma. The polarisation and the Gaussian beam shape should be preserved. Machining issues have to be taken into account. In order to fulfil these pre-conditions, pioneering work has been carried out at the University of Stuttgart $[5,6]$.

\subsection{Design properties of the gratings}

The required boundary conditions could be fulfilled with a $-3^{\text {rd }}$ order grating reflection and an efficiency $>90 \%$ is possible on average [5]. Fine structures in the grating 
profile serve to minimize the difference of efficiencies between the TE and the TM polarisation, e.g. figure 9.

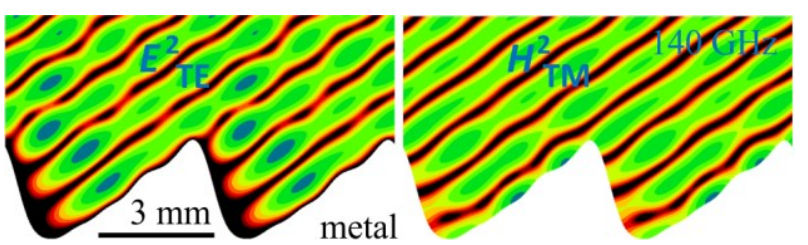

Fig. 9. Full wave simulation of the $140 \mathrm{GHz}$ mm-wave reflection on an optimized Fourier grating [5] with $\theta_{\mathrm{IN}}=60^{\circ}$, $\theta_{\mathrm{OUT}}=50^{\circ}$ and negative third order. Left: TE polarisation and calculated $89 \%$ efficiency for the reflection. Right: TM polarisation and $96.9 \%$ efficiency.

Each grating profile is optimized in the plane wave approximation. This corresponds to a local condition on the reflector. With a Gaussian beam shape, different positions on the reflector correspond to different optimized gratings. The surface structure of the full reflector is, therefore, interpolated from single optimized grating profiles and the phase front curvature of the Gaussian beam is taken into account by a curvature of the grooves of the grating [6]. The full reflector model is exemplarily shown in figure 10a and can be compared with the machined tile in figure $10 \mathrm{~b}$. The shown ' $\mathrm{R} 7$ ' reflector (for ECRH \#7) is now newly installed for the upcoming 2018 experimental campaign at AUG and its beam tracing design has been described elsewhere [10].

In the final design of each grating, an average beam direction and shape is used, which is the result of averaging the beam tracing in a set of different model plasmas. For the 'R7' reflector in figure 10, the average beam properties were characterised as follows:

$$
\begin{aligned}
\mathbf{k}_{\text {in }} & =[-0.51244,+0.30642,-0.80219] \\
\mathbf{k}_{\text {out }} & =[+0.49083,-0.48715,+0.72234]
\end{aligned}
$$

The angle between $\mathbf{k}_{\text {in }}$ and $\mathbf{k}_{\text {out }}$ is $\gamma=11.4^{\circ}$ and the coordinate system is like in figure $10 \mathrm{a}$ with $\mathrm{x}$-axis horizontal, y-axis in vertical direction towards the upper edge of the reflector, and $\mathrm{z}$-axis perpendicular to the reflector surface in the center. The Gaussian beam radii for both $\mathbf{k}_{\text {in }}$ and $\mathbf{k}_{\text {out }}$ are $w_{\mathrm{x}}=5.9 \mathrm{~cm}, w_{\mathrm{y}}=7.6 \mathrm{~cm}$, while the average data for the phase front curvature for the incoming beam are $R_{\mathrm{x}}=150 \mathrm{~cm}$ and $R_{\mathrm{y}}=128.5 \mathrm{~cm}$, both defocussing. For the reflected beam, the phase front curvature was designed with $R=-115 \mathrm{~cm}$ (both $x$ and $y$ ) and we note that the negative sign represents a focussing phase front. For each refocussing in the phase front curvature, a characteristic focal length $F$ can be introduced as in the following equation:

$$
R_{\text {out }}^{-1}=R_{\text {in }}^{-1}-F^{-1}
$$

This means that two characteristic focal lengths can be attributed to the grating 'R7': $F_{\mathrm{x}}=65.1 \mathrm{~cm}, F_{\mathrm{y}}=60.7 \mathrm{~cm}$.

\subsection{Laboratory measurements}

The reflecting grating ' $\mathrm{R} 7$ ' has been designed and optimized with fixed parameters for the incoming and reflected Gaussian beam. When the two-pass scheme operates with various plasma conditions, the actual values of the incoming Gaussian beam may deviate from the design values.


Fig. 10. Reflecting grating ' $R 7$ ' with the design (a) on top and the machined and tungsten coated tile (b) in the middle. The grating was machined at the workshop of the University of Stuttgart. The photo (c) at the bottom shows the rear part of the reflector with the thermocouple instrumentation, which has been done at IPP Garching. The darker area is due to the mounting of the tile during the $\mathrm{W}$ coating procedure.

It is therefore to be tested, if the characteristic focal lengths, as introduced in equation (2) can be used to model the effect of the grating in such case. For this purpose we made laboratory measurements of the 
outgoing phase front curvature, function of incoming phase front curvature, as shown in figure 11. Since in the 'R7' case the angle $\gamma$ between $\mathbf{k}_{\text {in }}$ and $\mathbf{k}_{\text {out }}$ is aligned nearly vertically, and since the reflection geometry on the laboratory table is horizontal, it should be noted that the original $\mathrm{x}$-axis (figure 10a) approximately corresponds to the vertical direction in the laboratory, and the original $y$-axis to the horizontal one.

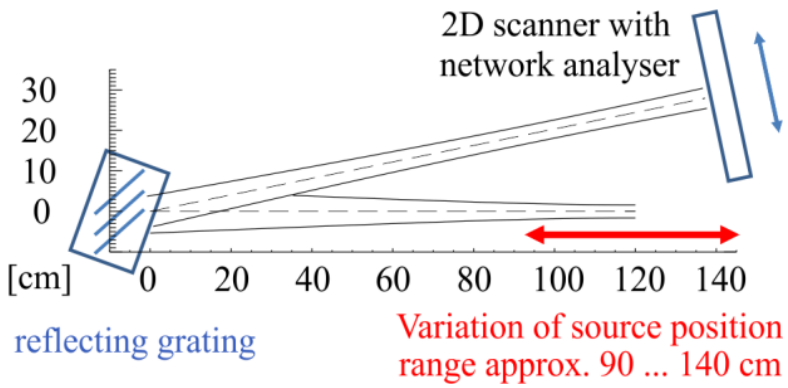

Fig. 11. Top view of the laboratory set-up for measuring the properties of the reflecting grating 'R7'. The source position was varied in order to change the incoming phase front curvature on the grating. For each source position, the reflected beam was scanned as indicated, which allowed to analyse the reflected beam. With these data the phase front curvature at the grating after the reflection could be determined.

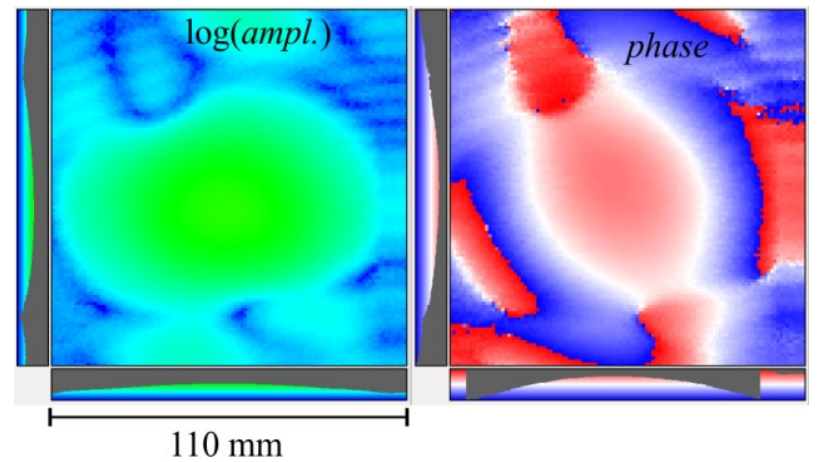

Fig. 12. Visualisation of amplitude (left, green: max, blue:min) and phase (right, colour coding $0 \ldots 2 \pi$, blue via white to red) of the reflected beam in the scanner plane, as described in figure 11. The small insets on the lower and left edges of each contour plot are cross sections through the center of the $2 \mathrm{D}$ plot. According to the numerical analysis, these data correspond to a Gaussian beam content of $96.6 \%$ with beam waists $w_{0}=1.41 \mathrm{~cm}$ (horizontal) and $1.56 \mathrm{~cm}$ (vertical). At the scanner position the beam is defocussing. The analysis yields $43.1 \mathrm{~cm}$ distance to the horizontal waist and $19.5 \mathrm{~cm}$ to the vertical beam waist. At the position of the grating this corresponds to phase front curvatures $-107.3 \mathrm{~cm}$ (horizontal) and $-132.7 \mathrm{~cm}$ (vertical) both focussing. The input phase front curvature was $139.2 \mathrm{~cm}$ (spherical, defocussing) at the grating.

The source $(140 \mathrm{GHz})$ position was varied between $90 \mathrm{~cm}$ and $140 \mathrm{~cm}$ (figure 11). Both polarisations were tested. The incoming phase front curvature varied approximately by the same amount, since the grating is already in the far field of the source. This variation is also typical for different experimental situations at AUG. For each measurement of the output beam parameters (e.g. figure 12) the equivalent focal lengths were deduced. With 9 different source positions, the deduced horizontal focal length (laboratory, corresponding to $F_{\mathrm{y}}$ ) was in the range $60.1 \ldots 61.3 \mathrm{~cm}$ and vertical in the range $66.9 \ldots 69.2 \mathrm{~cm}$. Due to the specific geometry and as shown in figure 12, the principal axes of the beam cross section are not perfectly aligned with the axes of the laboratory system. This contributes to the error in the data analysis. Altogether we consider these results reasonably consistent with the design (section 3.1). In the scanned area the measured Gaussian mode content of the reflected beam was always of the order $95 \%$.

The consistency of the set-up and the determination of the phase front curvature was verified with a spherical mirror with known focal length $F=80 \mathrm{~cm}$. The scan of the output beam showed a circular Gaussian beam with 98.6\% purity, and a change of the phase front curvature that corresponded to $F$ values of $77.4 \mathrm{~cm}$ (horizontal curvature) and $79.8 \mathrm{~cm}$ (vertical curvature), respectively.

Future measurements could take into account a shift of the beam with respect to the reflector center. Direct measurements of the total efficiency are highly desirable, but very demanding.

\section{Conclusions and Outlook}

With the development of ECRH O-2 schemes for high density, the necessary simulation tools and laboratory testing equipment is being developed in parallel. The design for AUG ECRH \#1-4 is being finalized and will make use of reflecting gratings. According to the presented measurement results, the characteristic focal lengths, attributed to each grating, seem to be adequate for modelling the reflection in the two-pass heating scheme. Focal lengths are free parameters and offer the possibility for future optimization.

The photos in figure 10 are a courtesy of Harald Schütz, IPP Garching. His careful assembly is gratefully acknowledged.

This work has been carried out within the framework of the EUROfusion Consortium and has received funding from the Euratom research and training programme 2014-2018 under grant agreement No 633053. The views and opinions expressed herein do not necessarily reflect those of the European Commission.

\section{References}

1. A. Kallenbach et al, Nucl. Fusion 55, 053026 (2015)

2. P. Lang et al, Nucl. Fusion 58, 036001 (2018)

3. V. Erckmann, U. Gasparino, Plasma Phys. Control. Fusion 36, 1869-1962 (1994)

4. M. Schubert et al, EPJ Web Conf 87, 02010 (2015)

5. O. Mangold, $\mathrm{PhD}$ thesis (Stuttgart University, 2009)

6. H. Höhnle et al, Nucl. Fusion 51, 083013 (2011)

7. D. Wagner et al, J Inf Milli Terahz 37, 45-54 (2016)

8. E. Poli et al, Comput. Phys Commun 136, 90 (2001)

9. M. Schubert et al, EPJ Web Conf 32, 02013 (2012)

10. M. Schubert et al, 43rd Conference Plasma Phys, ECA 40A, P1.026 (2016) 